\title{
Alteration of Symbiotic Microbiome Following the Decline of Korean Fir (Abies Koreana) and Its Relationship with Soil Environment in Regions of Mt. Hallasan, Jeju Island, the Republic of Korea
}

\author{
Minsoo Jeong \\ Kyungpook National University \\ Setu Bazie Tagele \\ Kyungpook National University \\ Min-Ji Kim \\ Kyungpook National University \\ Suk-Hyung Ko \\ World Heritage Office \\ Kwon-Su Kim \\ World Heritage Office \\ Jung-Goon Koh \\ World Heritage Office \\ Da-Ryung Jung \\ Kyungpook National University \\ YoungJae Jo \\ Kyungpook National University \\ YeonGyun Jung \\ Korea Institute of Oriental Medicine \\ Yeong-Jun Park \\ Kyungpook National University \\ Min-Sueng Kim \\ Kyungpook National University \\ Kyeongmo Lim \\ Kyungpook National University \\ Jae-Ho Shin ( $\nabla$ jhshin@knu.ac.kr) \\ Kyungpook National University
}

Research Article 
Keywords: Abies koreana, Forest decline, Microbiome, Symbiotic fungi, Mt. Hallasan, Jeju Island Posted Date: March 2nd, 2022

DOI: https://doi.org/10.21203/rs.3.rs-1338623/v1

License: (c) (1) This work is licensed under a Creative Commons Attribution 4.0 International License. Read Full License 


\section{Abstract \\ Background}

The Abies koreana (Korean fir) population is declining at an accelerating rate on Mt. Hallasan in Jeju Island, Republic of Korea. Several prior studies have reported various inconclusive reasons, indicating that additional data, such as data on microbial communities that promote plant growth and resistance to abiotic stresses, are required to further understand the phenomena. To the best of our knowledge, this is the first investigation that documents the changes in the microbial community as the result of the decline of the Korean fir forest. Here, high throughput sequencing data were provided for the rhizosphere microbiome of a Korean fir, identifying the bacterial and fungal composition differences between the rhizosphere soil of healthy Korean fir (HKF) and dead Korean fir (DKF).

\section{Results}

The results showed that the DKF soil of the fungal community was distinctly separated from the HKF soil. Interestingly, HKF soil comprised dominant symbiotic fungi (Russula, Sebacina, Inocybe, and Phenoliferia), whereas the microbial composition in DKF samples comprised abundant saprotrophs, such as Trichoderma, Ascocoryne, and Umbelopsis. It was also observed that symbiotic fungi, such as Russula, act as a hub in the co-occurrence network. Additionally, the representative fungal genera in HKF soil had a strong positive correlation with only a few soil physicochemical features: $\mathrm{K}^{+}, \mathrm{Na}^{+}, \mathrm{Avail} \mathrm{P}$, and cation exchange capacity (CEC).

\section{Conclusions}

Our findings shed light on the rhizosphere microbiota of dead and live A. koreana plants and their relationship with edaphic factors. The woody plant-associated symbiotic fungi, including ECMs, was also highlighted as a potential strategy to enhance the Korean fir forest ecosystem health.

\section{Background}

Korean fir, the scientific name for Abies koreana, is an evergreen tree known as a high-quality Christmas tree in demand for its special appearance and beautiful fragrance. The Korean fir grows only in subalpine southern regions at an altitude of 1,000-1,900 in the Republic of Korea, and Mt. Hallasan on Jeju Island is the world's largest habitat [1]. However, the Korean firs have faced serious decline and death for 20 years and were classified as Endangered (EN) in the IUCN 3.1 Red List of Threatened Species in 2011 [2].

Understanding the decline and dieback of the korean fir in Mt. Hallasan is one of the top research priorities in many research fields in Korea, which otherwise could cause the destruction of the natural 
balance of the mountain's plant ecosystem. Mt. Hallasan is the preserving site of several endemic tree species of the country. In previous studies, geographic, climatic, and soil physicochemical analyses, including tree density, solar radiation, terrain slope [3], and wind intensity changes [4], have been reported as factors contributing to the decline. However, there are still contrasting reports about the cause of the tree decline, and further studies remain to be conducted. Although findings diverge between studies; for example, Kang et al.[5] has attributed the decline to the decreased soil moisture content following increased temperature and insufficient winter snow cover, whereas Ahn et al.[3] has reported that excessive soil moisture caused by gradual terrain slope and decreased solar radiation contributed to the problem.

Furthermore, the soil microbiome plays a key role in ecosystem processing [6]. The microbial interaction, especially the rhizosphere microbiome, is complex and could lead to suppressive soil and induce tolerance to plants because of abiotic factors caused by climate change. The rhizosphere microbiome, in turn, depends on the plant genotype and environment [7]. More importantly, the rhizosphere microbiome of Korean fir has not yet been documented. Thus, this study assessed variations in the rhizosphere microbiome and soil physicochemical properties between HKF and DKF. The relationships between soil physicochemical qualities and microbiome were also assessed, and the potential contribution of the rhizosphere microbiome of Korean fir was investigated for induction of stress tolerance. For these reasons, differences between the soil microbiome structures of healthy Korean fir (HKF, $\mathrm{n}=20)$ and dead Korean fir (DKF, $\mathrm{n}=18$ ) sampled from the colony of Korean firs were investigated using $16 S$ rRNA and ITS2 amplicon sequencing. The correlation analysis between nutrients and soil microbes in the soil environment was also investigated using correlation analysis between the genera and soil physicochemical features, which varied significantly between the HKF and DKF groups. The cooccurrence network at the cross-kingdom level identified representative genera expected to have biological effects in the soil environment. Hence, the study aims to develop the current understanding of the Korean fir forest microbiome and provide a new biological perspective from the analysis of bacterial and fungal communities.

\section{Results}

\section{Soil physicochemical properties of the rhizosphere soil of Korean fir}

Twenty-eight soil physicochemical properties were measured for all samples collected from the Korean fir forest to compare differences between HKF and DKF soil properties (Table S1). Only five features among the 28 soil physicochemical features were significantly different $(p<0.05)$ between the two groups (Fig. 1). In the DKF soil samples, nutrient-related properties, including $\mathrm{K}^{+}, \mathrm{Na}^{+}$, and Avail $\mathrm{P}$, decreased. In addition, the soil CEC of DKF was low $(31.90 \pm 8.39)$ compared with HKF (38.81 \pm 7.42$)$. In contrast, the sand percentage of rhizosphere soil from DKF was high compared with HKF soil. No significant difference was observed in soil moisture content between the two groups. In the subsequent analysis, 
only five soil features showing significant differences between the HKF and DKF were used for the analysis.

\section{Microbiome community structure and diversity in HKF and DKF soil}

The bacterial and fungal community changes associated with the Korean fir forest decline were analyzed using the Next Generation Sequencing of 16S rRNA and ITS2 amplicons from the HKF and DKF rhizosphere soil samples. A total of 2,182 bacterial ASVs and 1,191 fungal ASVs were identified from 339,074 and 445,474 high-quality rarefied sequences of 38 soil samples (20 HKF and 18 DKF samples). From taxonomy-assigned ASVs, 29 bacterial phyla with 242 genera and ten fungal phyla with 257 genera were found. The top ten dominant phyla in each group were used for downstream analysis. A significant decrease in the phyla Basidiomycota and an increase in the phyla Ascomycota were found in DKF soil following the death of the Korean fir (Fig. 2A and 2B). However, no significant changes were found in the major bacterial phyla. The a-and $\beta$-diversity was also investigated to identify the differences in the microbial communities between the two groups at the ASV-level. The differences in relative abundance at the phylum level were also seen at the $\beta$-diversity analysis. PCoA based on the unweighted UniFrac distance of the soil microorganisms showed that bacterial communities were not statistically different (Fig. 2C). In contrast, the DKF cluster in fungal communities was significantly separated from the HKF cluster (Adonis, $p=7 e-04$ ) (Fig. 2D). Furthermore, the a-diversity measured by the Shannon-Wiener index and inverse Simpson reflecting the richness and evenness for the observed ASVs was analyzed (Fig. 2E and $2 \mathrm{~F}$ ). The fungal alpha diversity in the inverse Simpson index increased slightly in DKF compared with HKF, whereas no significant $(p=0.31)$ difference was found in the Shannon-Wiener index.

\section{Compositional differences in fungal communities}

As the fungal communities of HKF and DKF varied significantly $(p<0.001)$, the differential relative abundance of the mycobiome at the genus level was further investigated. Figure 3 indicates only genera with significant differences at a $25 \%$ prevalence threshold in relative abundance. Notably, Russula, Sebacina, and Phenoliferia, which are well known as symbiotic fungi (Table S2), were found only in the HKF group (Fig. 3A). Meanwhile, Trichoderma, Ascocoryne, and Umbelopsis were more abundant in the DKF group. In the analysis based on the fungal functional guide using FUNGuild (open fungal annotation tool, Guilds_v1.0), symbiotic and saprophytic fungi were predominantly observed in HKF and DKF groups, respectively (Fig. S1A). In HKF soil, the symbiotroph was more diverse on the Chao1 index and more abundant in relative abundance. The saprotroph diversity was slightly lower than in DKF soil (Fig. S1B). Furthermore, genus biomarkers with differential abundance between the HKF and DKF groups were identified using LEfSe analysis with a log (LDA score) over 3.0. In the HKF and DKF groups, 14 and 12 genera biomarkers, respectively, were yielded. In the LDA score of over 4, four genera, including Russula, Sebacina, and Inocybe, were enriched in the HKF group. In comparison, three genera, namely, Trichoderma, Leptodontidium, and Ascocoryne, were significantly higher in the DKF group (Fig. 3B).

\section{Network analysis of soil microorganisms}


Cross-kingdom network analysis was conducted for an overall understanding of the microbial matrix in the soil environment. To investigate the soil microbial relationship, co-occurrence networks were analyzed using Sparse InversE Covariance estimation for the Ecological Association and Statistical Inference (SPIEC-EASI). Genera with less than $25 \%$ occurrence or less than $0.1 \%$ relative abundance were estimated to decrease network noise and complexity. A total of 201 and 207 nodes, $90 \%$ of the initial genus relative abundance, were obtained across HKF and DKF samples, respectively. The networks of HKF and DKF groups consist of 437 and 415 edges, respectively. Transitivity $(T)$ and density (D) of the network were higher in HKF than in DKF (Fig. 4A and 4B). As a result of investigating the loss effect of the microbial community on network connectivity through the node-based random attack, the connectivity of the HKF network decreased more gradually than that of DKF, suggesting that HKF has a stronger and more complex network than DKF (Fig. 4C). We investigated the genera predicted to play a potentially important role in the network (Fig. 4D and 4E) through keystone taxa analysis based on the network betweenness centrality and node degree. Notably, while symbiotic fungi, such as ectomycorrhizal fungi Russula and Clavulina, were observed as keystone taxa in the HKF network, saprotroph fungi, such as Entoloma and Trichicadium, were mainly observed in the DKF network.

\section{Correlation between the soil mycobiome and physicochemical properties}

Correlation analysis was conducted between discriminative fungal taxa of the groups based on their relative abundance and LEfSe analysis. Soil physicochemical properties have indicated a significant difference between the groups and are more likely to affect the growth conditions of korean firs. The correlation analysis of the fungal genera indicated that certain genera that were significantly enriched in the HKF had a strong positive correlation with each other (Fig. 5). Notably, Russula, a genus that played a keystone role in the network, had statistically significant positive correlations with the most abundant fungal genera of HKF groups but not with DKF abundant fungal genera. The results of correlation analysis between soil physicochemical properties and fungal communities indicated that most genera had positive correlations with the heatmap in HKF groups. Russula, Phenoliferia, Krasilnikovozyme, and Leucosporidium correlated conspicuously with $\mathrm{K}^{+}$and $\mathrm{Na}^{+}$exchangeable cations. Only Sebacina positively correlated with Avail P. In the case of the genus in DKF, Hypochnicium and Leptosphaeria correlate negatively with the cation exchange capacity (CEC), and a weak correlation was generally observed.

\section{Discussion}

The decline of the Korean fir has long been a serious problem for which no clear cause has been found [8]. Most previous studies have reported a link between the tree decline and environmental factors, including typhoons [9], droughts [10], excess moisture [3, 11], and other climatic changes [12, 13]. Although soil microbiota plays an essential role in the forest ecosystem processes [14], the rhizosphere microbiome of Korean firs remains unknown. In addition, plant-associated soil microbial communities 
maintain soil health by stimulating plant growth, supplying nutrients, and increasing resistance to biological and abiotic stresses, such as climate change [15]. Thus, understanding the rhizosphere microbiota of healthy and dead plants of Korean firs and their association with edaphic factors will advance our knowledge about the contribution of microbial communities to resistance to abiotic stresses for such endangered tree species. It will also help in designing effective alternative strategies for conserving Korean firs. To the best of our knowledge, this study is the first report about the rhizosphere microbial structure of Korean firs. In addition, this study reports the association between microbiome and soil physicochemical features.

PCoA beta diversity and relative abundance analysis in our study showed that significant differences were observed between the two groups in the fungal community but not in the bacterial community. This result suggests that the fungal community assembly is comparatively more sensitive than the bacterial community to the stress-induced changes of the fir tree rhizosphere, emphasizing fungi as a good bioindicator of habitat transition [16]. In addition, the bacterial community response to abiotic factors in our study was similar to that reported in previous studies [17]. Bacteria have high plasticity to environmental stresses, which supports the idea that soil bacterial communities differ in their vulnerability to stresses [18] and host health status doesn't affect members of soil microbiota equally [19]. In addition to the direct impact of environmental stresses on microbial communities, the effect of changes in plant root exudates following abiotic stress on the microbial community cannot be ruled out [20].

The fungal diversity of the DKF soil sample was generally slightly higher than that of the HKF sample, which may be attributed to woody plants or crops manufacturing exudates to control an environment suitable for self-growth, which regulates symbiotic soil microorganisms through chemical interaction [21]. Alternatively, dead plant roots in DKF soil could create a conducive environment for different fungi groups, especially necrotroph microbes [21]. The proportion of symbiotic fungal genera in DKF soil was reduced, which might be attributed to the interaction between plant-derived metabolites and mycobiome [21]. Our study showed that although nonsignificant changes in bacterial diversity between the two groups were observed, fungal diversity appears to be comparatively more influenced by stress-induced rhizosphere changes in the Korean firs. However, more research is needed to confirm the evidence of such relationships.

Differential abundance, LEfSe, and co-occurrence network analysis at genus level between HKF and DKF showed that ectomycorrhizas (ECMs), including Russula, Sebacina, and Hydnotrya, had the highest abundance in the HKF group. Russula was also found to be a keystone taxa in the HKF network. Russula was found to be one of the most essential and abundant ECM fungi having a symbiotic relationship with diverse higher plants in the mountain rain forest, which conforms to the findings of previous studies [2225]. These keystone taxa in HKF play essential roles in the fungal communities of the soil environment. Interestingly, Kohout et al. [26] highlighted that ECMs, including Russula, mostly drove the rapid dynamics in the fungal community composition. In addition to Russula, a previous study has reported Sebacina as the commonly observed ECM in the forest ecosystem [27]. Hydnotrya has also been reported as one of 
the most dominant ECM symbiont fungal genera [28], which may be because of the presence of rhizodeposits from the live fir [26]. In contrast, there was a decrease in the abundance of such ECMs in dead plant roots [29], which might be attributed to the lack of plant-derived active carbon inputs, including exudates from the dead plant roots [30]. Co-occurrence network analysis also showed that HKF network, which has keystone taxa of known plant symbionts, is more stable and complex, and it has more efficient global connectivity than the DKF network, which conforms to previous studies that plants supply high nutrients to microbes, leading to more complex microbial networks [31].

Our soil physicochemical properties and correlation analysis indicate that discriminative symbiotic fungal taxa had a linear relationship with soil features. In DKF soil, the percentage of sand that has a large particle size and small surface area was relatively higher compared with HKF soil (Fig. 1). DKF also had reduced $\mathrm{CEC}, \mathrm{K}^{+}$, and $\mathrm{Na}^{+}$exchangeable cations, which have low adsorption strength to soil particles. $\mathrm{A}$ previous study had emphasized that ECM fungi can increase $\mathrm{CEC}, \mathrm{K}^{+}$, and $\mathrm{Na}^{+}$exchangeable cations in soil [40], which aligns with our findings that symbiotic fungi strongly correlate with soil nutrients (Fig. 5). Potassium and sodium are important nutrients for the forest ecosystem and defend against many biotic and abiotic stresses [32,33].

The soil of Jeju island is well known for its high phosphate absorption coefficient (Table S2) in Korea, which may be due to high ashes from volcano eruption [34]. Alternatively, although phosphate ( $\mathrm{Pi})$ is one of the important macronutrients for plant growth and survival [35], it has a strong affinity to bind to soil minerals and becomes unavailable to plants. In our results, high levels of Avail P in HKF soil may be partly attributed to the release of soluble phosphorus owing to dephosphorylation of the soil microbiome [36]. In line with this, a previous study had emphasized that Sebacina, which has a strong positive linear relationship with phosphate, has an acid phosphatase enzyme [37].

The high abundance of saprotrophs, such as Ascocoryne, Umbelopsis, and Hypochnicium, in the DKF group supports the fact that saprotrophs are found at very low abundance in actively growing plant roots [38], and they mainly feed on nonliving organic matter, such as dead plant roots [39]. Trichoderma spp., a key genus specializing in the breakdown of complex compounds, such as lignin and cellulose [40], which commonly arise after plant death [20], were likewise abundant in DKF soil. Collectively, these findings indicate a reduction in the abundance of symbiotic fungi in the rhizosphere of the declined tree population and an increase in saprotrophic fungi. Similar to previous studies, we found that different plant saprotrophic fungal genera act as network keystones in the DKF group [41]. Nevertheless, pathogenic fungal genera were not found to be as abundant in DKF soil as in HKF soil, which is consistent with previous findings that the survival of microbes, including ECMs, is related to soil nutrient availability.

\section{Conclusions}

Our results showed that the fungal diversity, especially ECMs, was more affected than bacterial diversity following the Korean fir forest decline. Although this is a baseline study about the rhizosphere 
microbiome of Korean firs, our research provides a new perspective on the phenomenon of such unknown fir forest decline. The use of plant-associated symbiotic fungi, including ECMs, was also highlighted as a potential strategy to enhance the Korean fir forest ecosystem health. However, large sample sizes are needed in future studies to fully address the cause of tree decline and understand the contribution of the Korean fir forest soil microbiome and ECMs in particular.

\section{Methods}

\section{Ethical clearance}

\section{Sample Location and physicochemical measurement}

All samples were obtained with site access and sampling collection permission from the World Heritage Office of Hallasan National Park. This study was conducted in Mt. Hallasan National Park, Jeju-si, Jeju Province, Jeju Island, Republic of Korea $\left(33^{\circ} 21^{\prime} \mathrm{N}, 126^{\circ} 31^{\prime} \mathrm{E}\right)$. A total of 60 soil samples were obtained from fir forest distribution areas from October 2020. We selected 38 samples (20 soil samples of HKF and 18 of DKF) to remove possible noise from categorical response variables such as sampling sites. (Fig. 6). Rhizospheric soil was acquired from the ground firmly attached to the roots of Korean firs. Samples for measuring physical properties were collected using a DIK-1630 soil picker (DAIKI, Saitama, Japan). Samples for measuring chemical properties were collected while considering the layer after removing the organic layer at the same point where the physical samples were collected. Soil physicochemical analysis was performed through routine methods [42]. The samples for measuring chemical features were taken after removing the organic layer at the same point where the samples were obtained. Soil samples were air-dried and passed through a 2-mm sieve for chemical analysis. The samples were used for soil physicochemical analysis, including $\mathrm{pH}\left(\mathrm{H}_{2} \mathrm{O}, \mathrm{CaCl}_{2}, \mathrm{KCl}, \mathrm{NaF}\right)$, electrical conductivity $(E C)$, organic matter(OM), total nitrogen ( $(\mathrm{N}-\mathrm{N})$, available phosphate (Avail P), phosphate absorption coefficient, exchangeable cations $\left(\mathrm{K}^{+}, \mathrm{Ca}^{2+}, \mathrm{Mg}^{2+}, \mathrm{Na}^{+}, \mathrm{Al}^{3+}\right)$, soil texture (Sand, Silt, Clay), particle density, bulk density, porosity, soil three phase distribution and hydraulic conductivity. Soil samples for microbial DNA analysis were frozen at $-70^{\circ} \mathrm{C}$ without air-drying and stored in polyethylene sample bottles.

\section{Soil bacterial and fungal DNA extraction, amplification, and sequencing}

Soil microbial DNA extraction was performed using the DNeasy PowerSoil Pro Kit (Qiagen, Hilden, Germany) according to the manufacturer's protocols. The extracted DNA was quantified based on the DNA absorbance ratio at 260/280 nm ( 1.9) and 260/230 nm ( 2.0) using the NanoDrop One UV-Vis spectrophotometer (Thermo Fisher Scientific, Waltham, MA, USA). The DNA concentration was remeasured for accuracy using a Qubit 2.0 Fluorometer (Thermo Fisher Scientific, Waltham, MA, USA). After DNA extraction, the prokaryotic $16 S$ rRNA V4 - V5 hypervariable region was amplified through primer pair 515F/907R (515F, 5'- ACA CTC TTT CCC TAC ACG ACG CTC TTC CGA TCT GTG NCA GCB GCC GCG 
GTR A -3' and 907R, 5'- GTG ACT GGA GTT CAG ACG TGT GCT CTT CCG ATC CGY CWA TTY HTT TRA GTT T -3') [43, 44], and the eukaryotic ITS2 region was amplified through primer pair ITS86F/ITS4R (ITS86F, 5'-ACA CTC TTT CCC TAC ACG ACG CTC TTC CGA TCT GTG AAT CAT CGA ATC TTT GAA-3' and ITS4R, 5'-GTG ACT GGA GTT CAG ACG TGT GCT CTT CCG ATC TCC TCC GCT TAT TGA TAT GC-3') [45, 46]. For the polymerase chain reaction (PCR) to amplify purified DNA, 25-mL EmeraldAmp MAX HS PCR Master Mix (Takara Bio, Shiga, Japan), 2-mL extracted DNA, 1-mL each primer, and 21-mL sterile distilled water were used in a 50-mL reaction using Eppendorf Mastercycler Nexus PCR Cycler (Eppendorf, Hamburg, Germany). For the prokaryotic $16 S \operatorname{SRNA}$, a denaturation step of $95^{\circ} \mathrm{C}$ was performed for 3 min, followed by 20 cycles at $95^{\circ} \mathrm{C}$ for $30 \mathrm{~s}, 56^{\circ} \mathrm{C}$ for $30 \mathrm{~s}$, and $72^{\circ} \mathrm{C}$ for $30 \mathrm{~s}$, and a final extension at $72^{\circ} \mathrm{C}$ for 3 min. For the eukaryotic Internal Transcribed Spacer (ITS) 2 region, a denaturation step of $95^{\circ} \mathrm{C}$ was performed for $5 \mathrm{~min}$, followed by 30 cycles at $95^{\circ} \mathrm{C}$ for $30 \mathrm{~s}, 58^{\circ} \mathrm{C}$ for $30 \mathrm{~s}$, and $72^{\circ} \mathrm{C}$ for $30 \mathrm{~s}$, and a final extension at $72^{\circ} \mathrm{C}$ for $5 \mathrm{~min}$. The second PCR process for attaching the dual multiplexing index and specific adaptor was as follows: for the prokaryotic $16 \mathrm{~S} r R N A$, a denaturation step of $95^{\circ} \mathrm{C}$ was performed for $3 \mathrm{~min}$, followed by 25 cycles at $95^{\circ} \mathrm{C}$ for $30 \mathrm{~s}, 56^{\circ} \mathrm{C}$ for $30 \mathrm{~s}$, and $72^{\circ} \mathrm{C}$ for $30 \mathrm{~s}$, and a final extension at $72^{\circ} \mathrm{C}$ for $3 \mathrm{~min}$. For the eukaryotic d region, a denaturation step of $95^{\circ} \mathrm{C}$ was performed for $5 \mathrm{~min}$, followed by 30 cycles at $95^{\circ} \mathrm{C}$ for $30 \mathrm{~s}, 58^{\circ} \mathrm{C}$ for $30 \mathrm{~s}$, and $72^{\circ} \mathrm{C}$ for $30 \mathrm{~s}$, and a final extension at $72^{\circ} \mathrm{C}$ for 5 min. The size and quality of the amplicon were verified through $2 \%(w / v)$ agarose gel electrophoresis, and amplicon product impurities were eliminated using QIAquick Gel Extraction Kit (Qiagen, Hilden, Germany). The amplified and purified DNA amplicon was adjusted to the same DNA concentration and pooled considering the PCR product size of the 16S rRNA and ITS2. Paired-end sequencing was performed using the Illumina Miseq instrument (Illumina, San Diego, USA) with adjustable read lengths of about $2 \times 250$ bp using Miseq Reagent Kit v3 (Illumina, 600 cycles). Fastq files have been deposited into an NCBI Sequence Read Archive (SRA, NCBI, http://www.ncbi.nlm.nih.gov/sra) under the accession numbers PRJNA802974. Sequencing was performed at the next-generation core facility of Kyungpook National University.

\section{Bioinformatics}

The demultiplexed raw fastq data acquired from the Miseq instrument were filtered for quality and analyzed using the Quantitative Insights Into Microbial Ecology 2 (QIIME2) (ver 2020.11) software [47]. Since the merged paired sequences are mostly filtered owing to low-quality reverse reads, only forward sequences were used for subsequent analysis. To generate amplicon sequence variants (ASVs), the single-end sequence was filtered (mean frequency; 26,260 for bacteria, and 37,312 for fungi), trimmed (with lengths of 200 nucleotides), and denoized (Q score threshold > 30) using the DADA2 software [48]. The ASVs were assigned taxonomy with a $99 \%$ cutoff value by the classify-sklearn program using the Silva 16S/18S rRNA full-length sequences (Release 138) [49] and UNITE database (ver 2019.02) [50] as the reference database. The ASVs classified as mitochondria, chloroplast, eukaryote, or unassigned were eliminated from the bacterial ones, and the ASVs classified as mitochondria, prokaryote, or unassigned were removed from the fungal ones. Alignment was conducted using de novo multiple sequence alignment with an align-to-tree-mafft-fasttree plugin of QIIME2. The sequences were rarefied (with a depth of 8,923 reads for bacteria, 11,723 reads for fungi). 


\section{Statistical analysis}

Statistical analysis and visualization were conducted using the R software (ver 4.0.3). All QIIME2 data inputs to R software were processed after pre-processing using the "Phyloseq" R package (ver 1.34.0) and the "qiime2R" R package (ver 0.99.4). The amplicon sequencing result and statistical significance of soil physicochemical qualities were evaluated using the unpaired two-sample t-test, Welch's correction paired t-test, and Wilcoxon rank-sum test. The normality test was performed using the Shapiro-test function in the "stat" R package (ver 4.0.3), and the test of equal variances was performed with the Levene-test function in the "car" R package (ver 3.0-10) for two independent groups. Shannon, InvSimpson, Chao1, and Evenness indices for alpha diversity analysis were computed using the "vegan" R package. Beta diversity was conducted to evaluate differences between groups using principal coordinates analysis ( $\mathrm{PCOA}$ ) based on Bray-Curtis dissimilarity and unweighted UniFrac distance. Permutational multivariate analysis of variance was conducted using an Adonis test from the "vegan" R package. To investigate the biomarkers characterizing the differences in the microbial communities of tree soil in different health states, linear discriminant analysis effect size (LEfSe) [51] was performed using the "microbiomeMarker" $\mathrm{R}$ package (ver 0.0 .1 .9000 ). Correlation analysis of the matrix between genus biomarker and soil physicochemical qualities within each group was conducted using Spearman's rho rank correlation coefficients with the "Hmisc" R package (ver 4.4-2).

\section{Microbial network analysis}

Inter-kingdom co-occurrence network analysis in HKF and DKF soil samples was performed using the Sparse InversE Covariance estimation for the Ecological Association and Statistical Inference (SpiecEasi, ver 1.1.0, https://github.com/zdk123/SpiecEasi) R package. To reduce complexity and noise in the network, genera with a relative abundance of less than $70 \%$ in the whole or less than ten reads in each sample were excluded. The sparse graphical lasso was used. For the HKF soil microbial network, 116 bacterial and 85 fungal ASVs were used, whereas 122 bacterial and 85 fungal ASVs were used for the DKF network.

\section{Abbreviations}

HKF : Healthy Korean fir

DKF : Dead Korean fir

Avail P : Available phosphate

CEC : Cation exchange capacity

EC : Electrical conductivity

OM : Organic matter 
T-N : Total nitrogen

ASV : Amplicon sequence variants

PCoA : Principal coordinates analysis

FUNGuild : Fungal functional guide

LEfSe : Linear discriminant analysis effect size

LDA : Linear Discriminant Analysis

SPIEC-EASI : Sparse InversE Covariance estimation for the Ecological Association and Statistical Inference

ECMs : Ectomycorrhizas

$\mathrm{Pi}$ : Phosphate

ITS : Internal Transcribed Spacer

PCR : Polymerase chain reaction

QIIME2 : Quantitative Insights Into Microbial Ecology 2

EN : Endangered

IUCN : International Union for Conservation of Nature

\section{Declarations}

\section{Code availability}

All codes used in this work are available upon request to the authors.

\section{Ethics approval and consent to participate}

Not applicable

\section{Availability of data and materials}

All raw sequences (fastq files) have been deposited into an NCBI Sequence Read Archive (SRA, NCBI, http://www.ncbi.nlm.nih.gov/sra) under the accession numbers PRJNA802974. 


\section{Consent for publication}

Not applicable

\section{Competing interests}

The authors declare that they have no competing interests

\section{Funding}

This paper was written as a result of the analysis of soil microorganisms in the native area of the fir in Mt. Hallasan, and was supported by the Ministry of Environment and Jeju Special Self-Governing Province, and we are grateful for this. We also thanks to the project to train professional personnel in biological materials by the Ministry of Environment, South Korea.

\section{Authors' contributions}

All authors contributed to the study conception and design. Conceived and designed the experiments: MJ, YGJ, SHK, KSK, JGK and JHS. Performed the experiments: MJ, SHK, KSK, JGK, YGJ, YJJ, YJP, MSK and KL. Analyzed the data: MJ, MJK, DRJ, YJP. Wrote the paper: MJ, SBT and JHS. All authors wrote, commented, and accepted the final version of the manuscript.

\section{Corresponding author}

Correspondence to Jae-Ho Shin

\section{Acknowledgements}

We express our thank the support and cooperation of the KNU NGS core facility regarding microbiome sequencing.

\section{Authors' information}

\section{Affiliations}

Department of Applied Biosciences, Kyungpook National University, Daegu 41566, Republic of Korea 
Minsoo Jeong, Setu Bazie Tagele, Min-Ji Kim, Young Jae Jo, Yeong-Jun Park, Min-Sueng Kim, Kyeongmo Lim \& Jae-Ho Shin

Hallasan Research Department, World Heritage Office, Jeju Special Self-Governing Province, 63143, Republic of Korea

Suk-Hyung Ko, Kwon-Su Kim \& Jung-Goon Koh

Department of Biomedical Convergence Science \& Technology, Kyungpook National University, Daegu 41566, Republic of Korea

Da-Ryung Jung \& Jae-Ho Shin

Korean Medicine (KM)-Application Center, Korea Institute of Oriental Medicine, 70 Cheomdan-ro, Dong-gu, Daegu 41062, Republic of Korea

YeonGyun Jung

\section{References}

1. Ihm BS, Lee JS, Kim MH, Kim HS. A Comparative Study on the Habitat of Abies koreana Wilson between Mt. Jiri and Mt. Hallasan. Plant Resources. 2000;3(2):138-147.

2. Kim YS, Chang CS, Kim CS, Gardner M. Abies koreana. The IUCN Red List of Threatened Species 2011: e.T31244A9618913. 2011. https://dx.doi.org/10.2305/IUCN.UK.2011-

2.RLTS.T31244A9618913.en. Accessed on 20 December 2021.

3. Ahn US, Yun YS. Causes of Decline in the Korean Fir Based on Spatial Distribution in the Mt. Hallasan Region in Korea: A Meta-Analysis. Forests. 2020;11(4):391. https://doi.org/10.3390/f11040391

4. Song KM, Kim J, Kang YJ, Choi HS, Jung SC, Lee S Y, Kim CS (2016) Korea Fir Hallasan Mountain, Why Are They Dying. National Institute of Forest Service. 2016. p. 136

5. Kang SJ. Regeneration process of subalpine coniferous forest in Mt. Jiri. Korean J Ecology. 1984;7(4):185-193.

6. Dubey A, Malla MA, Khan F, Chowdhary K, Yadav S, Kumar A, Khan ML. Soil microbiome: a key player for conservation of soil health under changing climate. Biodivers Conserv. 2019;28(8):2405-2429. https://doi.org/10.1007/s10531-019-01760-5

7. Kawasaki A, Donn S, Ryan PR, Mathesius U, Devilla R, Jones,A, Watt M. Microbiome and exudates of the root and rhizosphere of Brachypodium distachyon, a model for wheat. PloS One. 2016;11(10) e0164533. https://doi.org/10.1371/journal.pone.0164533

8. Seo JW, Choi EB, Park JH, Kim YJ, Lim HI. The Role of Aging and Wind in Inducing Death and/or Growth Reduction in Korean Fir (Abies Koreana Wilson) on Mt. Hallasan, Korea. Atmosphere. 2021;12(9):1135. https://doi.org/10.3390/atmos12091135 
9. Kim JK, Koh JG, Yim HT, Kim DS. Changes of Spatial Distribution of Korean fir Forest in Mt. Hallasan for the Past 10 Years(2006, 2015). Korean J Ecology. 2017;31(6):549-556.

https://doi.org/10.13047/KJEE.2017.31.6.549

10. Kim NS, Han D, Cha JY, Park YS, Cho HJ, Kwon HJ, Cho YC, Oh SH, Lee CS. A Detection of Novel Habitats of Abies Koreana by Using Species Distribution Models(SDMs) and Its Application for Plant Conservation. Korean J Ecology. 2015;18(6):135-149.

https://doi.org/10.13087/kosert.2015.18.6.135

11. Ahn US, Kim DS, Yun YS, Ko SH, Kim KS, Cho IS. The inference about the cause of death of Korean Fir in Mt. Hallasan through the analysis of spatial dying pattern - Proposing the possibility of excess soilmoisture by climate changes -. Korean J Agric For Meteorol. 2019;21(1):1-28. https://doi.org/10.5532/KJAFM.2019.21.1.1

12. Koo KA, Kim DB. Review Forty-year Studies of Korean fir(Abies koreana Wilson). Korean J Ecology. 2020;34(5):358-371. https://doi.org/10.13047/KJEE.2020.34.5.358

13. Kim ES, Oh CH, Park HC, Lee SH, Choi J, Lee SH, Yoon YK. Disturbed regeneration of saplings of Korean fir (Abies koreana Wilson) an endemic tree species, in Hallasan National Park, a UNESCO Biosphere Reserve, Jeju Island, Korea. J Mar Isl. 2016;5(1):68-78. https://doi.org/10.1016/j.imic.2016.02.001

14. Lladó S, López-Mondéjar R, Baldrian P. Forest soil bacteria: diversity, involvement in ecosystem processes, and response to global change. Microbiol Mol Biol Rev. 2017;81(2) e00063-16. https://doi.org/10.1128/MMBR.00063-16

15. Berg G, Köberl M, Rybakova D, Müller H, Grosch R, Smalla K. Plant microbial diversity is suggested as the key to future biocontrol and health trends. FEMS Microbiol Ecol. 2017;93(5) https://doi.org/10.1093/femsec/fix050

16. Bai Y, Wang Q, Liao K, Jian Z, Zhao C, Qu J. Fungal community as a bioindicator to reflect anthropogenic activities in a river ecosystem. Front Microbiol. 2018;9:3152. https://doi.org/10.3389/fmicb.2018.03152

17. Justice SS, Hunstad DA, Cegelski L, Hultgren SJ. Morphological plasticity as a bacterial survival strategy. Nat Rev Microbiol. 2008;6(2):162-168. https://doi.org/10.1038/nrmicro1820

18. Classen AT, Sundqvist MK, Henning JA, Newman GS, Moore JA, Cregger MA, Patterson CM. Direct and indirect effects of climate change on soil microbial and soil microbial-plant interactions: What lies ahead?. Ecosphere. 2015;6(8):1-21. https://doi.org/10.1890/ES15-00217.1

19. Preiswerk D, Walser JC, Ebert D. Temporal dynamics of microbiota before and after host death. ISME J. 2018;12(8):2076-2085. https://doi.org/10.1038/s41396-018-0157-2

20. Williams A, de Vries FT. Plant root exudation under drought: implications for ecosystem functioning. New Phytol. 2020;225(5):1899-1905. https://doi.org/10.1111/nph.16223

21. Prudence SM, Newitt JT, Worsley SF, Macey MC, Murrell JC, Lehtovirta-Morley LE, Hutchings MI. Soil, senescence and exudate utilisation: Characterisation of the Paragon var. spring bread wheat root microbiome. Environ microbiome. 2021;16(1):1-21. https://doi.org/10.1186/s40793-021-00381-2 
22. Bzdyk RM, Olchowik J, Studnicki M, Nowakowska JA, Oszako T, Urban A, Hilszczańska D. Ectomycorrhizal colonisation in declining oak stands on the Krotoszyn Plateau, Poland. Forests. 2019;10(1):30. https://doi.org/10.3390/f10010030

23. Wang P, Zhang Y, Mi F, Tang X, He X, Cao Y, Xu J. Recent advances in population genetics of ectomycorrhizal mushrooms Russula spp. Mycology. 2015;6(2):110-120. https://doi.org/10.1080/21501203.2015.1062810

24. Izumi H. Temporal and spacial dynamics of metabolically active bacteria associated with ectomycorrhizal roots of Betula pubescens. Biol Fertil Soils. 2019;55(8):777-788. https://doi.org/10.1007/s00374-019-01393-4

25. Ważny R, Kowalski S. Ectomycorrhizal fungal communities of silver-fir seedlings regenerating in fir stands and larch forecrops. Trees. 2017;31(3) 929-939. https://doi.org/10.1007/s00468-016-1518-y

26. Kohout $P$, Charvátová $M$, Štursová $M$, Mašínová $T$, Tomšovský $M$, Baldrian P. Clearcutting alters decomposition processes and initiates complex restructuring of fungal communities in soil and tree roots. ISME J. 2018;12(3):692-703. https://doi.org/10.1038/s41396-017-0027-3

27. Long D, Liu J, Han Q, Wang X, Huang J. Ectomycorrhizal fungal communities associated with Populus simonii and Pinus tabuliformis in the hilly-gully region of the Loess Plateau, China. Sci Rep. 2016;6(1):1-10. https://doi.org/10.1038/srep24336

28. Leski T, Rudawska M. Ectomycorrhizal fungal community of naturally regenerated European larch (Larix decidua) seedlings. Symbiosis. 2012;56(2) 45-53. https://doi.org/10.1007/s13199-012-01644

29. Wei F, Feng H, Zhang D, Feng Z, Zhao L, Zhang Y, Xu X. Composition of rhizosphere microbial communities associated with healthy and Verticillium wilt diseased cotton plants. Front microbiol. 2021;12. https://doi.org/10.3389/fmicb.2021.618169

30. Yu F, Liang JF, Song J, Wang SK, Lu JK. Bacterial community selection of Russula griseocarnosa mycosphere soil. Front microbiol. 2020;11:347. https://doi.org/10.3389/fmicb.2020.00347

31. Yang Y, Shi Y, Kerfahi D, Ogwu MC, Wang J, Dong K, Adams JM. Elevation-related climate trends dominate fungal co-occurrence network structure and the abundance of keystone taxa on Mt. Norikura, Japan. Sci Total Environ. 2021;799:149368.

https://doi.org/10.1016/j.scitotenv.2021.149368

32. Glowa KR, Arocena JM, Massicotte HB. Properties of soils influenced by ectomycorrhizal fungi in hybrid spruce [Picea glaucax engelmannii (Moench.) Voss]. Can J Soil Sci. 2004;84(1):91-102. https://doi.org/10.4141/S03-031

33. Wang $\mathrm{M}$, Zheng $\mathrm{Q}$, Shen $\mathrm{Q}$, Guo $\mathrm{S}$. The critical role of potassium in plant stress response. Int $\mathrm{J}$ Mol Sci. 2013;14(4):7370-7390. https://doi.org/10.3390/ijms14047370

34. Ryu IS. Comparison between phosphorus absorption coefficient and Langmuir adsorption maximum. Korean J Soil Sci Fert. 1975;8(1):1-17.

35. Tripler CE, Kaushal SS, Likens GE, Todd Walter M. Patterns in potassium dynamics in forest ecosystems. Ecol Lett. 2006;9(4):451-466. https://doi.org/10.1111/j.1461-0248.2006.00891.x 
36. Havlin JL. FERTILITY. Encyclopedia of Soils in the Environment. Elsevier. 2005. p. 10-19.

37. Malla R, Pokharel U, Prasad R, Varma A. Molecular Techniques to Study Polymorphism between Closely Related Microorganisms in Relation to Specific Protein Phosphatase. Springer, Berlin, Heidelberg, 2010. p. 339-361.

38. Zhu N, Zhu Y, Kan Z, Li B, Cao Y, Jin H. Effects of two-stage microbial inoculation on organic carbon turnover and fungal community succession during co-composting of cattle manure and rice straw. Bioresour Technol. 2021;341:125842. https://doi.org/10.1016/j.biortech.2021.125842

39. Wei F, Feng H, Zhang D, Feng Z, Zhao L, Zhang Y, Xu X. Composition of rhizosphere microbial communities associated with healthy and Verticillium wilt diseased cotton plants. Front Microbiol. 2021;12. https://doi.org/10.3389/fmicb.2021.618169

40. Pusz W, Baturo-Cieśniewska A, Kaczmarek-Pieńczewska A, Patejuk K, Czarnota P. Is the Survivability of Silver Fir under Condition of Strong Ungulate Pressure Related to Mycobiota of Bark-Stripping Wounds?. Forests. 2021;12(8):976. https://doi.org/10.3390/f12080976

41. Münzenberger B, Golldack J, Ullrich A, Schmincke B, Hüttl RF. Abundance, diversity, and vitality of mycorrhizae of Scots pine (Pinus sylvestris L.) in lignite recultivation sites. Mycorrhiza. 2004;14(3):193-202. https://doi.org/10.1007/s00572-003-0257-2

42. NIAST (National Institute of Agricultural Science and Technology). Method of soil and plant analysis. Rural Development Administration. Suwon, Korea; 2010

43. Apprill A, McNally S, Parsons R, Weber L. Minor revision to V4 region SSU rRNA 806R gene primer greatly increases detection of SAR11 bacterioplankton. Aquat Microb Ecol. 2015;75(2):129-137. DOI: https://doi.org/10.3354/ame01753

44. Parada AE, Needham DM, Fuhrman JA. Every base matters: assessing small subunit rRNA primers for marine microbiomes with mock communities, time series and global field samples. Environ Microbiol. 2016;18(5):1403-1414. https://doi.org/10.1111/1462-2920.13023

45. Ferrer C, Colom F, Frasés S, Mulet E, Abad JL, Alió JL. Detection and identification of fungal pathogens by PCR and by ITS2 and 5.8 S ribosomal DNA typing in ocular infections. J Clin Microbiol. 2001;39(8):2873-2879. https://doi.org/10.1128/JCM.39.8.2873-2879.2001

46. Turenne CY, Sanche SE, Hoban DJ, Karlowsky JA, Kabani AM. Rapid identification of fungi by using the ITS2 genetic region and an automated fluorescent capillary electrophoresis system. J Clin Microbiol. 1999;37(6):1846-1851. https://doi.org/10.1128/JCM.37.6.1846-1851.1999

47. Bolyen E, Rideout JR, Dillon MR, Bokulich NA, Abnet CC, Al-Ghalith GA, Caporaso JG. Reproducible, interactive, scalable and extensible microbiome data science using QIIME 2. Nat Biotechnol. 2019;37(8):852-857. https://doi.org/10.1038/s41587-019-0209-9

48. Callahan BJ, McMurdie PJ, Rosen MJ, Han AW, Johnson AJA, Holmes SP. DADA2: high-resolution sample inference from Illumina amplicon data. Nature methods. 2016;13(7) 581-583. https://doi.org/10.1038/nmeth.3869

49. Quast C, Pruesse E, Yilmaz P, Gerken J, Schweer T, Yarza P, Glöckner FO. The SILVA ribosomal RNA gene database project: improved data processing and web-based tools. Nucleic Acids Res. 2012; 
41(D1):D590-D596. https://doi.org/10.1093/nar/gks1219

50. Abarenkov K, Henrik Nilsson R, Larsson KH, Alexander IJ, Eberhardt U, Erland S, Kõljalg U. The UNITE database for molecular identification of fungi-recent updates and future perspectives. New Phytol. 2010;186(2):281-285. https://doi.org/10.1111/j.1469-8137.2009.03160.x

51. Segata N, Izard J, Waldron L, Gevers D, Miropolsky L, Garrett WS, Huttenhower C. Metagenomic biomarker discovery and explanation. Genome Biol. 2011;12(6):1-18. https://doi.org/10.1186/gb2011-12-6-r60

\section{Figures}

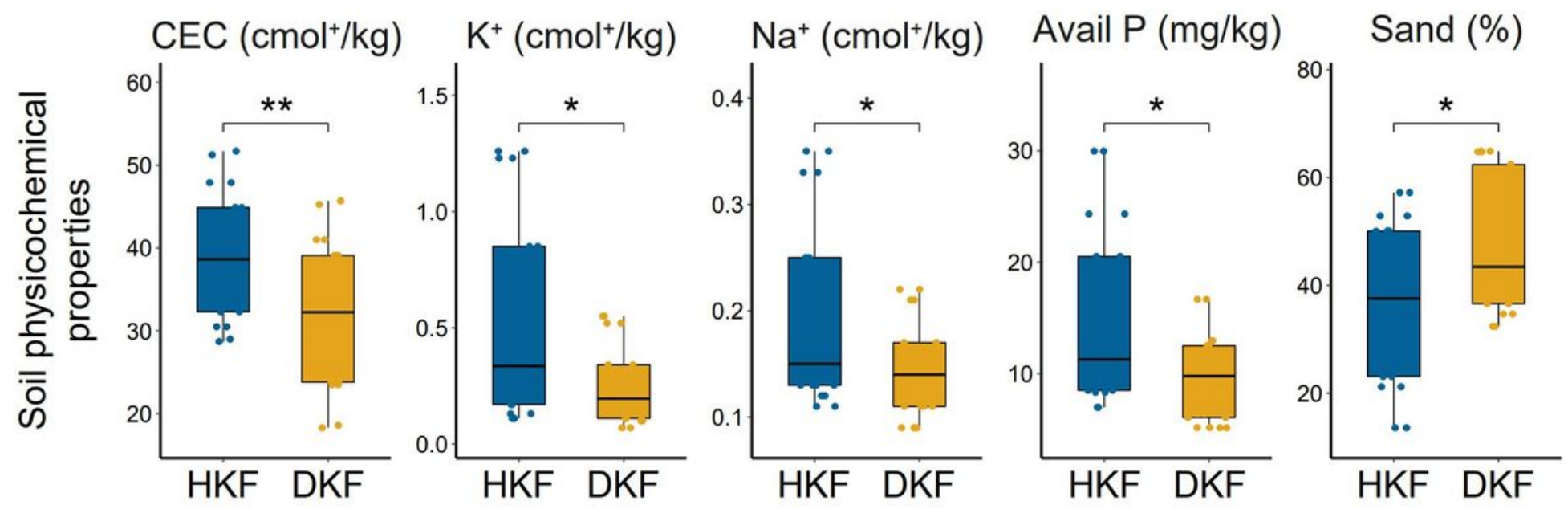

Figure 1

Comparison of soil physicochemical properties between soil samples. The Wilcoxon rank-sum test was used to estimate statistical significance; ${ }^{\star} p<0.05,{ }^{*} p<0.01$. HKF, healthy Korean fir; DKF, dead Korean fir; CEC, cation exchange capacity. 
a

Bacterial Phylum
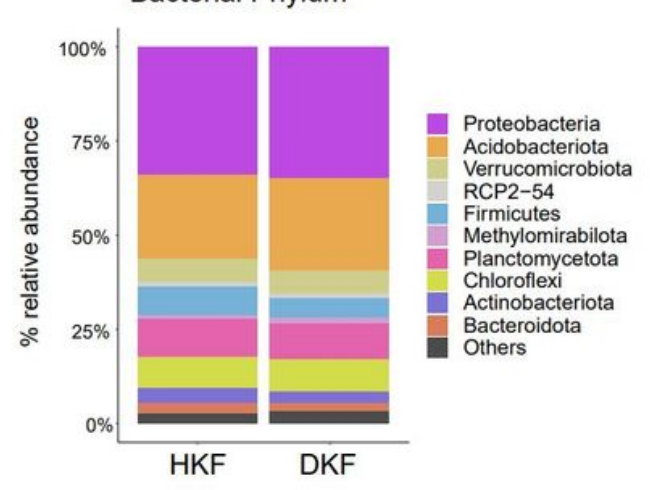

b

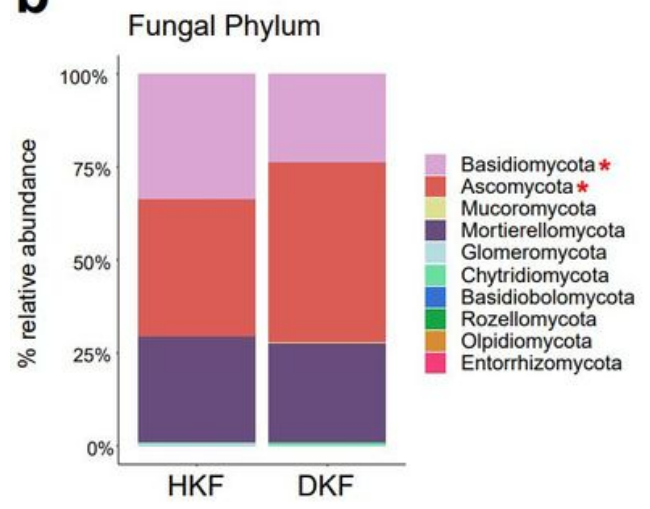

C

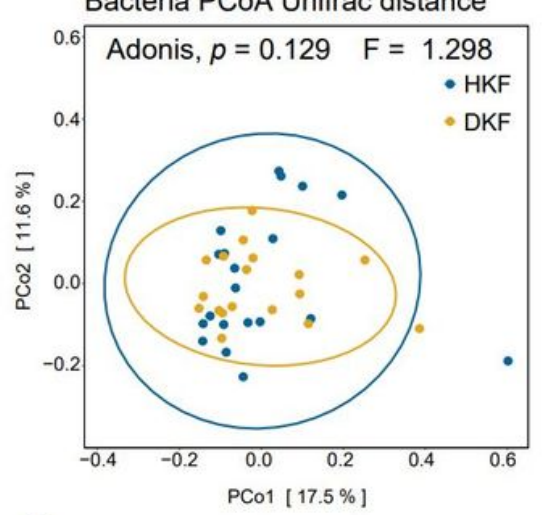

d

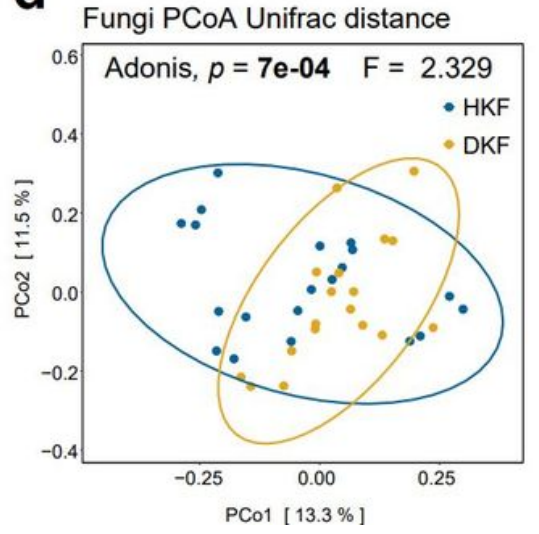

e
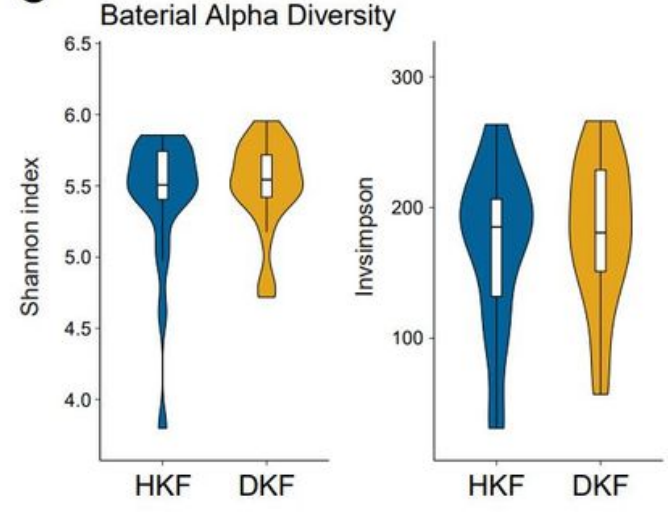

f

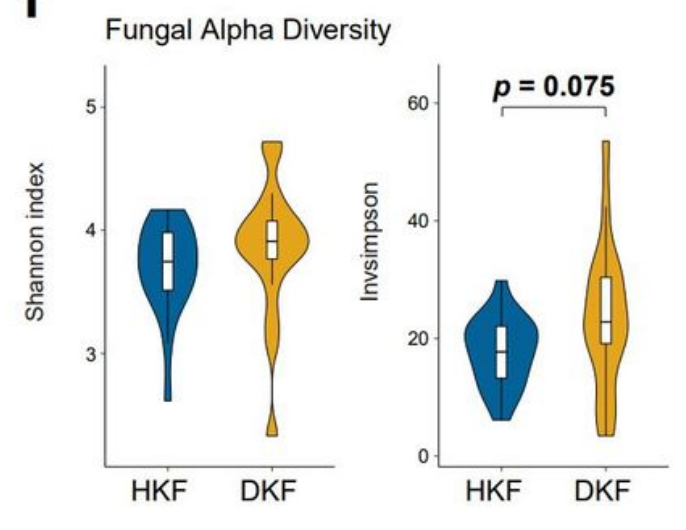

Figure 2

A comparison of the microbiome community structure and diversity between the rhizospheric samples.

The upper part is the analysis of the bacterial community, and the lower part is the analysis of the fungal community. (A) Relative abundance of top ten dominant phyla in each group. Other phyla are included as "Others." (B) Principal coordinates analysis (PCoA) based on unweighted UniFrac distance and microbial diversity differences between the HKF (blue) and DKF (yellow) groups ( $p<0.05$, Adonis). (C) Shannon and inverse Simpson diversity index of Korean fir samples between two separate health conditions. 
a
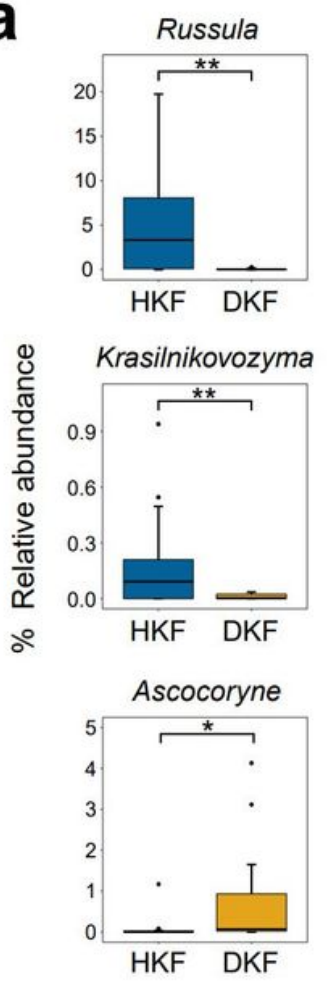
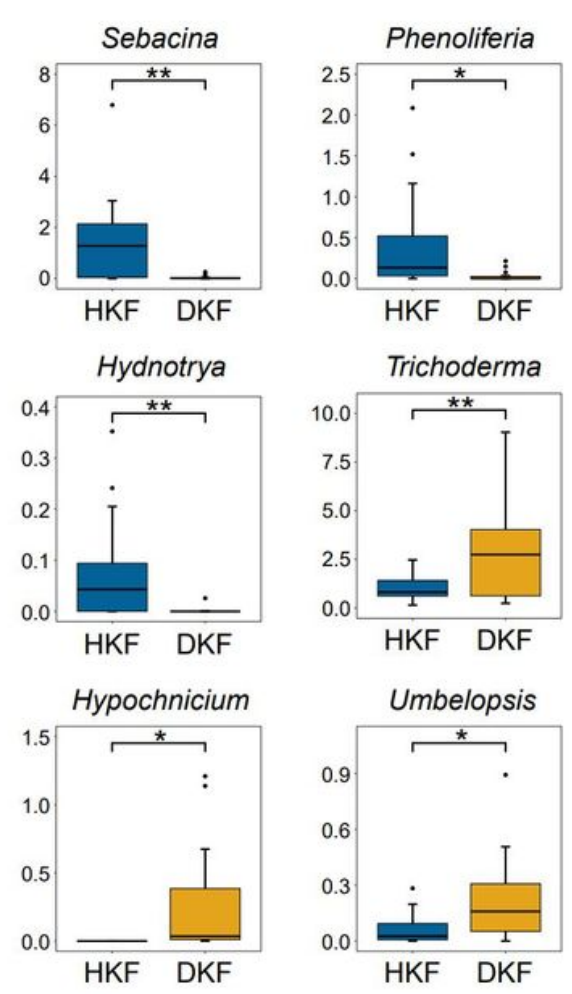

b

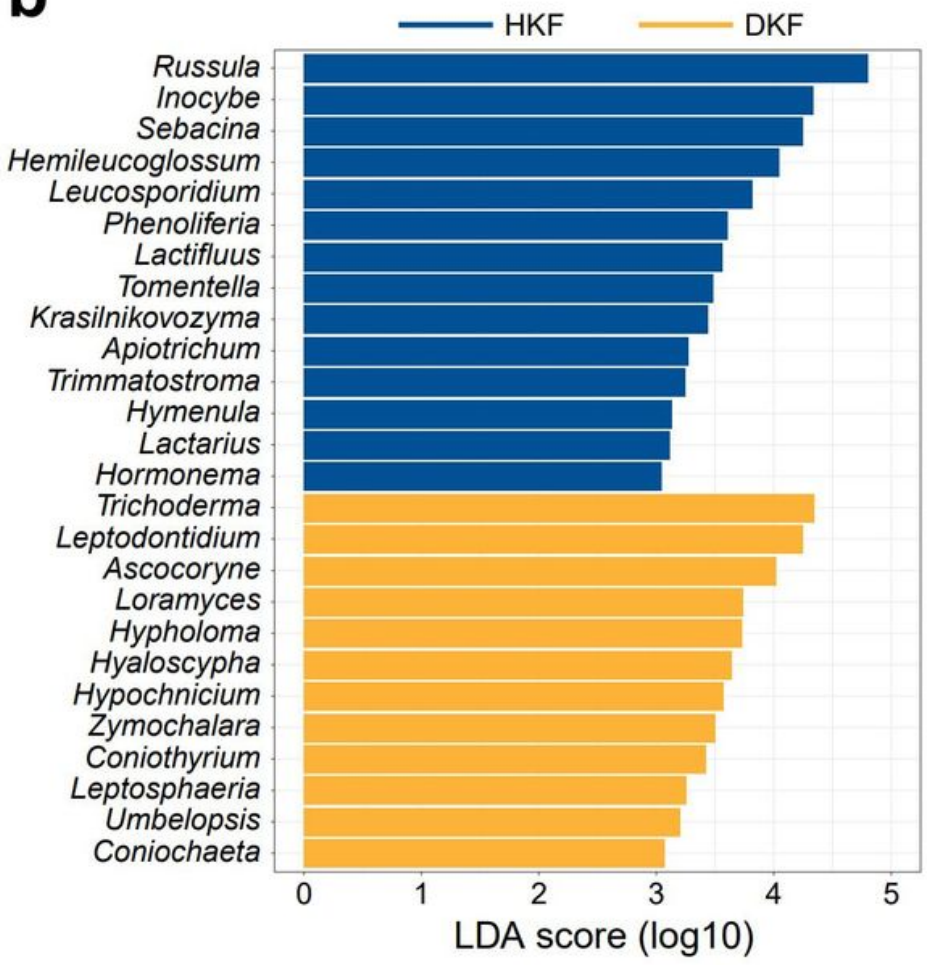

Zymochalara

eptosphaeria Umbelopsis

LDA score $(\log 10)$

\section{Figure 3}

Representative biomarkers at fungal genera level. (A) Box plots represent the relative abundance of fungal genera observed in the HKF and DKF groups. Differential abundance analysis presents only genera with significant differences $\left({ }^{\star} p<0.05,{ }^{\star \star} p<0.01\right)$. (B) linear discriminant analysis effect size analysis based on LDA score to detect biomarkers indicating differences between the groups (with LDA score $>3.0$ ) 
a

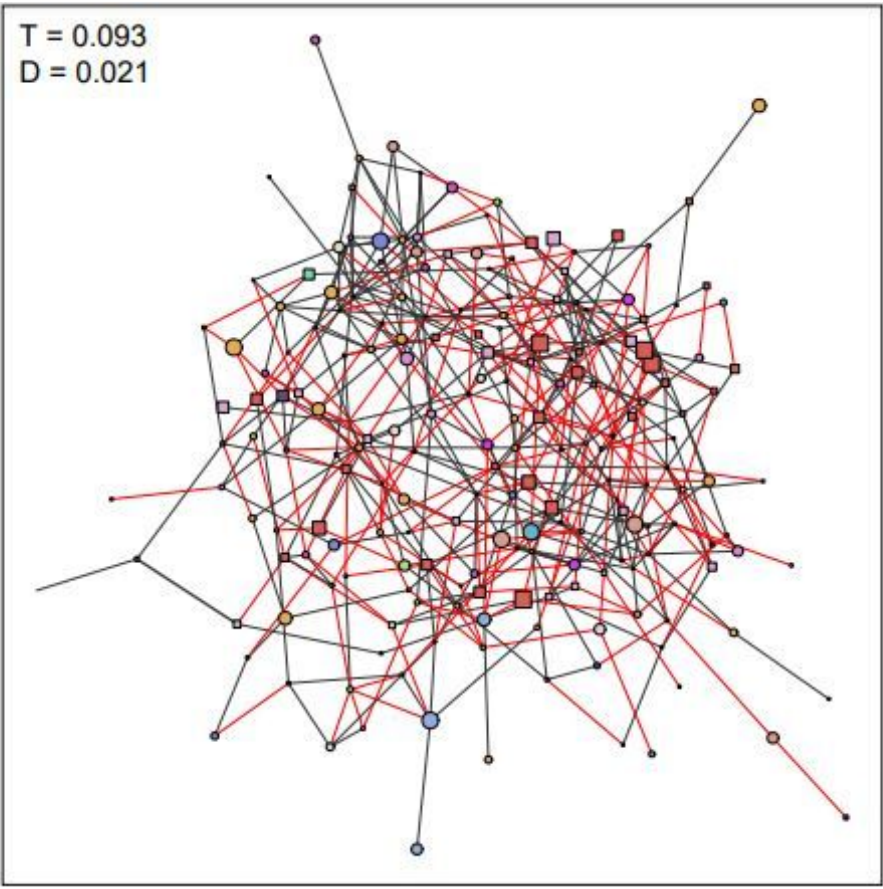

b

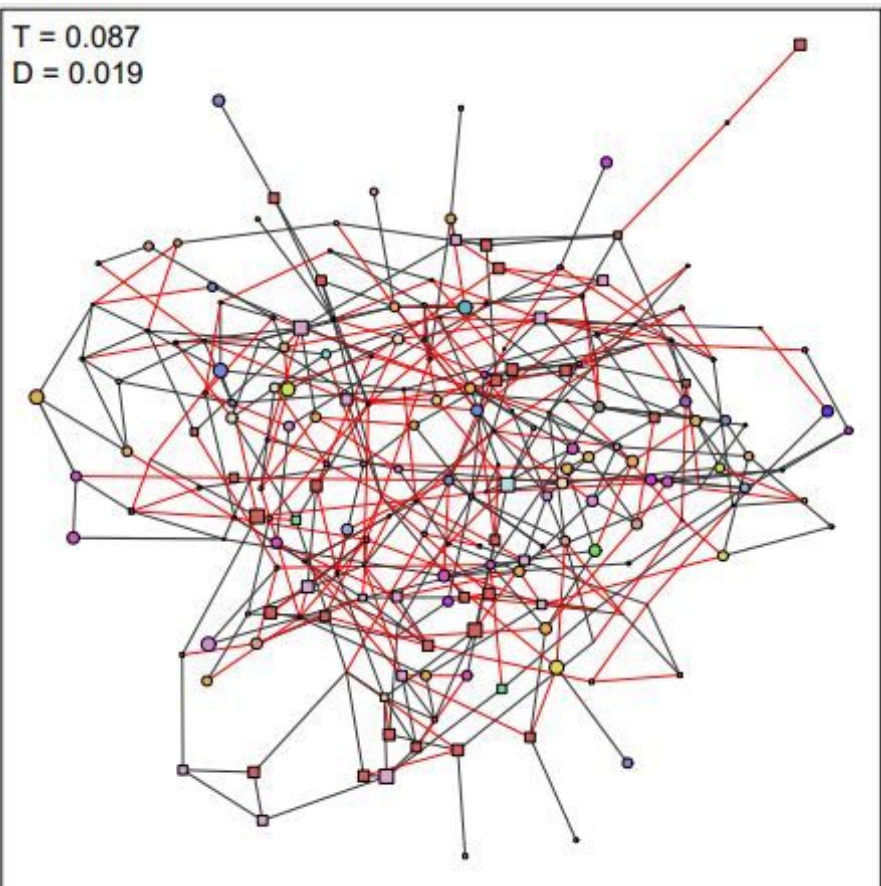

C

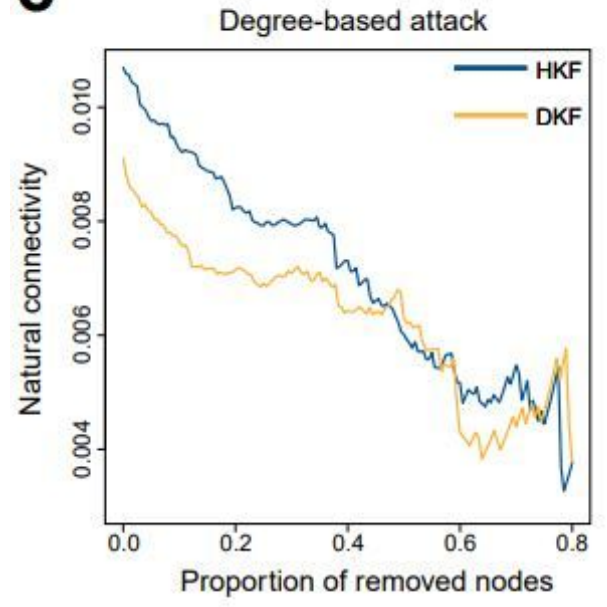

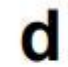

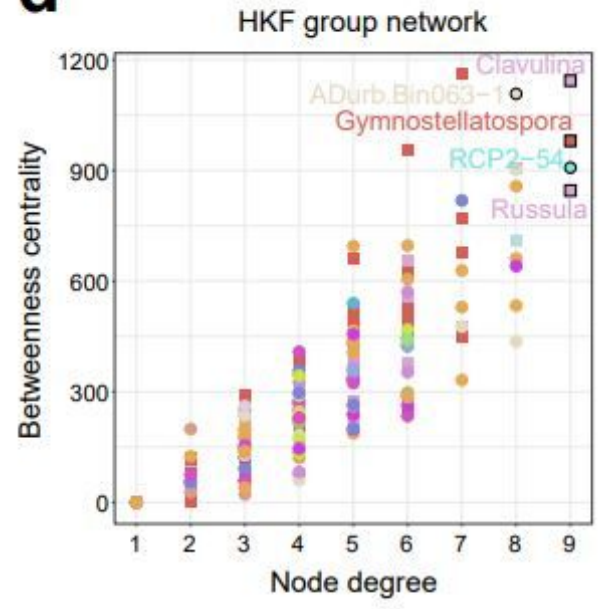

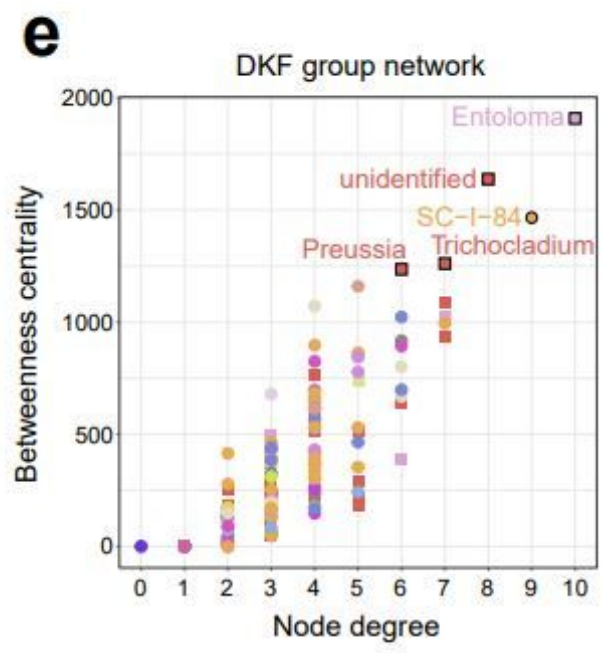

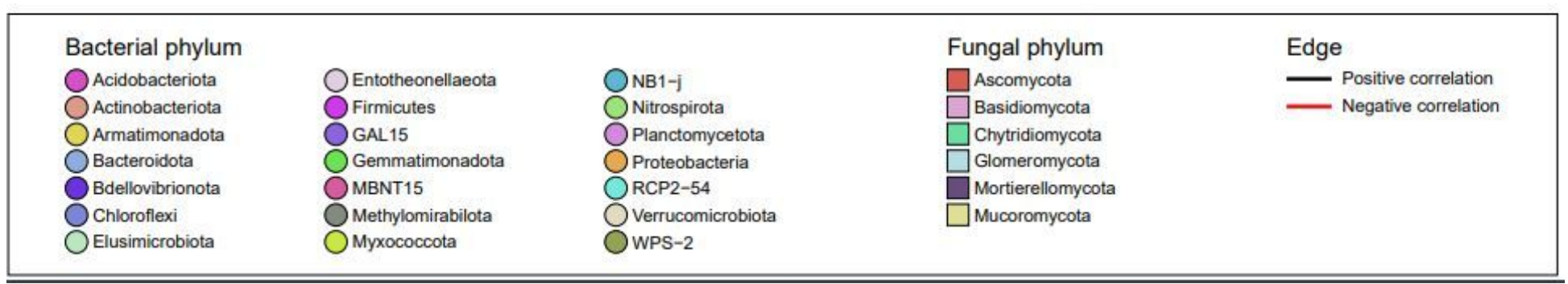

\section{Figure 4}

Cross-kingdom co-occurrence correlation network analysis of soil microbiome. Cross-kingdom network structure for the healthy Korean fir (HKF) (A) and dead Korean fir (DKF) (B) groups. The relative abundance of taxa was represented through the node size, kingdom division through the node shape (bacteria in circle, fungi in square), and genera through the node color. Positive and negative correlations were indicated as black and red edges, respectively. The edge thickness shows correlation strength. (C) 
Attack robustness curves measure the node percentage in the connected component by eliminating nodes based on betweenness. (D, E) Keystone taxa analysis shows a possible essential role in the network based on the network betweenness centrality and node degree.

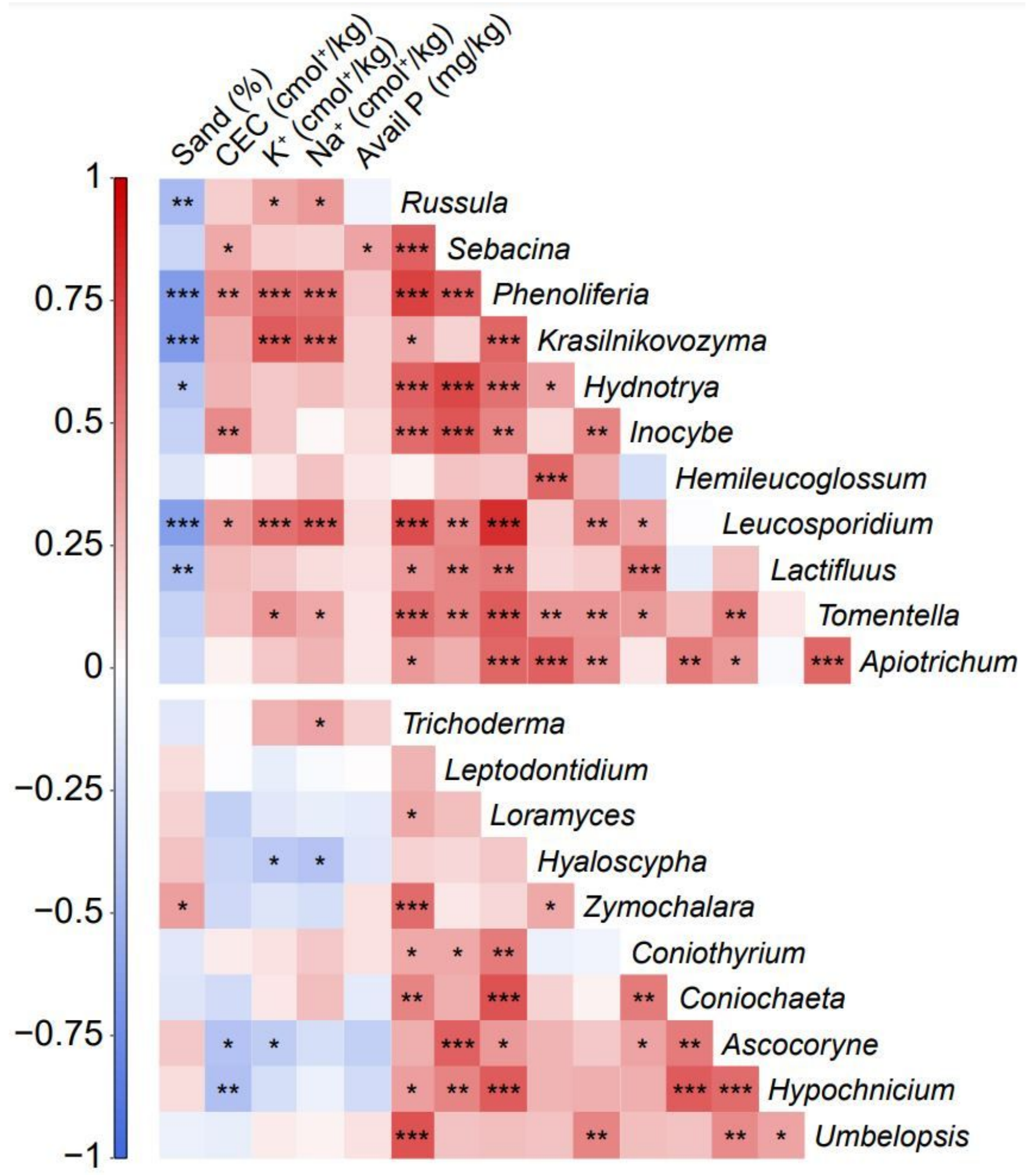

Figure 5 
Correlation heatmap results between discriminative taxa within fungal communities and soil physicochemical features. Red indicates a positive correlation, whereas blue exhibits a negative correlation using Spearman's rho rank correlation coefficients. Deeper color denotes greater correlation $\left({ }^{*} p<0.05,{ }^{*} p<0.01, * \star * p<0.001\right)$.

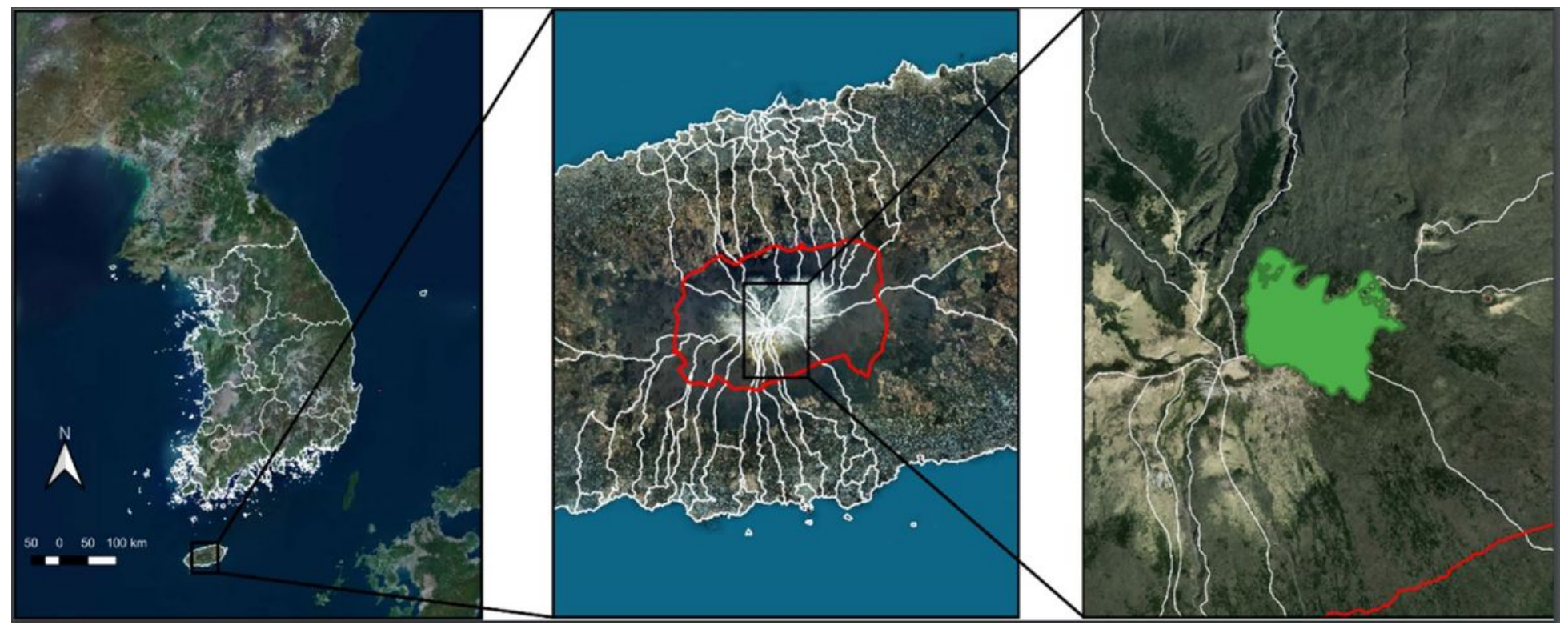

\section{Figure 6}

Sampling site of the Korean fir forest. The sampling site is a Korean fir colony (green) near Baengnokdam Crater Lake, the summit of Mt. Hallasan. The boundaries of Mt. Hallasan National Park are marked in red.

\section{Supplementary Files}

This is a list of supplementary files associated with this preprint. Click to download.

- Additionalfile1.docx 